\title{
Biological Model : Caenorhabditis elegans
}

\author{
Gitanjali Devi* \\ Department of Nematology, Assam Agricultural University, Jorhat-13, Assam, India \\ *Corresponding author
}

\section{Keywords \\ Biological model, Caenorhabditis elegans, developmental biology \\ Article Info \\ Accepted: \\ 12 April 2021 \\ Available Online: \\ 10 May 2021}

\section{Introduction}

A model organism is a biological but nonhuman species that is commonly studied by the scientists to realize some biological phenomena, with the probability that discoveries made in the organism model will provide insight into the workings of other organisms. Model organisms are in vivo models and are widely used to research human disease when human testing would be unfeasible or unethical (Muller and Grossniklaus, 2010).Most model organisms share a set of common characters that make them amenable to study in the laboratory. They are generally small, easy, and economical to culture in the lab, and reproduce quickly and can be manipulated in the laboratory. The best genetic model organisms have small genome sizes, and many can reproduce sexually, allowing researchers to cross-breed individuals of different genotypes, and have short generation time. These organisms can undergo mutation in their DNA that may result in a change in a certain characteristic, and these mutants allow scientists to study certain diseases. Examples of model organisms used to study genetics are yeast (Saccharomyces cerevisiae), fruit fly (Drosophila melanogaster), nematode (Caenorhaditis elegans), western clawed frog (Xenopus tropicalis), mouse (Mus musculus), and zebrafish (Danio rerio). The nematode, Caenorhabditis elegans shares a common ancestor with humans that lived in the preCambrian era, i.e. 500-600 million years ago. This ancestor is also known as the urbilaterian ancestor, as it is the relative of bilaterally 
symmetric, multicellular organisms on the planet, including invertebrates and vertebrates. Most of the genes and genetic mechanisms that govern modern organismal development, including those involved in human development and disease were present in the urbilaterian ancestor and are shared by existing animals, including humans and nematodes.

\section{Caenorhabditis elegans as biological model}

Caenorhabditis elegans is one of the most intensively used model organisms in biological studies. It is a multicellular eukaryotic organism under family: Rhabditidae, Order: Rhabditida, class: Chromodorea, Phylum: Nematoda, Kingdom: Animalia. C.elegans, is a free-living nematode inhabiting organic matter rich environments like rotting fruits and stems in many parts of the world. In 1900, Maupas named it Rhabditides elegans; in 1952, Osche placed it in the subgenus Caenorhabditis and in 1955, Dougherty raised it to the status of genus. The different Caenorhabditis species, C. briggsae, $C$. remanei, $C$. japonica and $C$. brenneri occupy various nutrient and bacteria-rich environments.

Key features of this nematode include its small body size, anatomical simplicity (>1000 cells), easy to culture, short life cycle, large size of progeny, cheap to maintain, transparent, invariant cell lineage, small genome (only 20 times that of Escherichia coli), and present no biohazard.

The virtues of $C$. elegans for genetic analysis include that it is small size $(1.5 \mathrm{~mm}$ long adult), easily grown in the laboratory on agar plates, on non-pathogenic Escherichia coli, reproduces quickly to produce approximately 300 offspring within a reproductive cycle of only 3.5 days at room temperature. The natural $C$. elegans mode of inbreeding by the self-fertilizing hermaphrodite combined with the ability to cross hermaphrodites with males offered advantages, in which crossing can be manipulated at will. C.elegans lacks a respiratory and a circulatory system. C.elegans has a simple but sophisticated nervous system (White et al., 1986). In the hermaphrodite, comprise 302 neurons, whose pattern of connectivity, also known as a connectome (neuronal 'wiring diagram') has been completely mapped out in the year 2012 . Researchers have explored the neural mechanisms responsible for several interesting behaviors including chemotaxis, thermotaxis, mechano transduction, and mating behavior. C. elegans possesses gut granules in the intestine which emit a brilliant blue fluorescence. Recent chemical analysis has identified the blue fluorescent material contain as a glycosylated form of anthranilic acid (AA), which is derived from tryptophan by action of the kynurenine pathway. AA is antibacterial and used in defense against invading pathogens and also provides photo protection (the bursts of AA fluorescence involve the conversion of damaging UV light to relatively harmless visible light).This compound can also serve a role as a natural, endogenous marker of organism death (Coburn and Gems, 2013).

The early work of Doughtery and associates provided the concepts and methods of axenic and monoxenic cultures of $C$. elegans (Ferris and Hieb, 2015). The initiation of the research using $C$. elegans by Sidney Brenner in the 1960 s resulted in a large number of worldwide research projects on this nematode. In 1963, Sydney Brenner proposed research into $C$. elegans primarily in the area of neuronal development; the ultimate goal was to determine the role of each gene involved in neural development and function (Chalfie et al., 1985). In 1974, he began research into the molecular and developmental biology of $C$. elegans, which has since been extensively 
used as a model organism. C. elegans was the first multicellular organism to have its whole genome sequenced. The project initiated by Sulston and associates to construct a physical map of the entire genome of $C$. elegans in the mid 1980s and the publication of the complete genome of C. elegans in 1998 contributed much to opening genomics as a discipline (Sulston et al.,1992; Bird et al.,1999). The genome contains an estimated 20,470 proteincoding genes.

The resulting genome sequence of $C$. elegans is an invaluable resource for the study of plant and animal parasitic nematodes. About $35 \%$ of C. elegans genes have human homologs-many C. elegans genes can function similarly to mammalian gene. In 2003, the genome sequence of the related nematode $C$. briggsae $C$. remanei, $C$. japonica and $C$. brenneri was also determined, allowing researchers to study the comparative genomics of these organisms.

It was initially proposed as a model for developmental biology because of its invariant body plan, ease of genetic manipulation and low cost of maintenance. Nematodes have affixed, genetically determined number of cells, a phenomenon known as eutely and the constancy of cell position from individual to individual have perhaps been the most unique advantages offered by this organism for the study of development. C.elegans is transparent, facilitating the study of cellular differentiation and other developmental processes in the intact organism. The developmental fate of every single somatic cell (959 in the hermaphrodite; 1031 in the male) has been mapped out. These somatic cells in a hermaphrodite represent a wide variety of tissues from all three germ layers, and the developmental biology of C. elegans has been traced from zygote to adult. In both sexes, a large number of additional cells (131 in hermaphrodite, most of which would otherwise become neurons) are eliminated by programmed cell death (apoptosis). By studying apoptosis in C.elegans, researchers hope to identify genes that switch on cell death in cancer cells, such as leukemia. C.elegans has proved to be a very useful model organism for investigating the roles of cell signaling and induction in development. Signals from neighboring cells direct gene expression and development (induction), and cell migrations around developing embryo. The related genetics in developmental biology including vulva formation, the dauer larvae pathway, programmed cell death, and underlying biochemistry apply to insects, mammals (including man), as well as nematodes and likely other organisms (Sommer and Bumbarger, 2012). Moreover, extensive research on C. elegans has identified RNA-binding proteins as essential factors during germ line and early embryonic development. Sulston's delineating cell lineage and the role of cell death in $C$. elegans and Horvitz's identifying a number of genes that control this programmed cell death brought new insight on the developmental biology of that nematode and all of its cells.

C. elegans has been a model organism for research into ageing (Rodriguez et al., 2013; Shen et al., 2018). A number of genes are responsible for longevity and senescence in nematodes have been identified (Denzel et al., 2019).

A useful feature of C.elegans is that the function of specific genes can be disrupted by RNA interference (RNAi). Silencing the function of a gene in this way can sometimes allow a researcher to infer what the function of that gene may be. The nematode can either be soaked in or injected with a solution of double stranded RNA, the sequence of which is complementary to the sequence of the gene that the researcher wishes to disable. RNA interference (RNAi) in C.elegans can also be done by simply feeding the nematode 
transgenic bacteria expressing RNA complementary to the gene of interest. This strategy for gene loss of function experiments is the easiest of all animal models, and thus, scientists are able to knock down $86 \%$ of the 20,000 genes in the worm, establishing a functional role for $9 \%$ of the genome (Darryl et al., 2015).

Brenner also chose C.elegans as it is easy to grow and can be frozen. When subsequently thawed they remain viable, allowing long-term storage. A $15 \%$ glycerol solution is used for the freezing of C.elegans. Samples are cooled at $1^{0} \mathrm{C}$ per minute. Freshly starved young larvae survive freezing best. About 35 to $45 \%$ of the worms stored in liquid nitrogen survive. The worms can also be stored at $-80^{\circ} \mathrm{C}$ for over ten years, but survival is not as great as for worms stored (25 years) in liquid nitrogen at $-196^{\circ} \mathrm{C}$ (Mikus et al.,2016).

C. elegans used as a model system to elucidate the toxicology, as well as, in environmental toxicology. $C$. elegans genetics clearly provides an advantage in toxicology tests as toxin effects can be directly linked to gene activities allowing for determination of sublethal effects (Martinez-Finley and Aschner, 2011). Studies have been primarily concerned with finding lethal endpoints of metals and organic compounds in single species cultures, aqueous solution, soil and sediment dose bioassays (Sochova et al.,2006). An exciting recent application of the C. elegans genetic model is to address ecology and evolution questions of broad concern to biologists. Examples of applications include a Yersinia pestis - C. elegans model to show biofilm-mediated interactions between bacteria and predatory invertebrates (Darby et al., 2002), the advantage of sexual reproduction to increase developmental flexibility (male or hermaphrodite) of progeny under changing resource conditions (Prahlad et al.,2003), that starvation stress induces adult diapause as a means of survival and dispersion (Angelo and Van Gilst, 2009; Kim et al.,2019) and modification of foraging strategies in response to environmental conditions (Boender et al.,2011).Other examples of recent use of the $C$. elegans model include showing that growth at high densities resulted in genetic changes to pheromone receptors (McGrath et al.,2011) and that sexual reproduction in $C$. elegans allows coevolution to survive against the pathogen Serratia marcescens (Morran et al.,2011). Even though, C. elegans is a suitable model for multiscale analysis from molecular to organism/population level, the range of $C$. elegans studies in environmental toxicology have been focused mostly on organism-level endpoints, such as mortality behavior, growth, or reproduction.

In 2002, the Nobel Prize in Physiology or Medicine was awarded to Sydney Brenner, $\mathrm{H}$. Robert Horvitz, and John Sulston for their work on the genetics of organ development and programmed cell death in $C$. elegans. The 2006 Nobel Prize in Physiology or Medicine was awarded to Andrew Fire and Craig C. Mello for their discovery of RNA interference in C. elegans. In 2008, Martin Chalfie shared a Nobel Prize in Chemistry for his work on green fluorescent protein; some of the research involved the use of $C$. elegans. C. elegans made news when specimens were discovered to have survived the Space Shuttle Columbia disaster in February 2003. Later, in January 2009, live samples of $C$. elegans from the University of Nottingham were announced to be spending two weeks on the International Space Station, in a space research project to explore the effects of zero gravity on muscle development and physiology. Descendants of the nematode aboard Columbia in 2003 were launched into space on Endeavour for the STS-134 mission. Although these works does not include plant and soil nematology, it brings much attention to nematodes. 
The early and ongoing research achievements on the model nematode $C$. elegans have provided a valuable resource for biology and genetics. While the $C$. elegans model now serves as an immense resource for investigating systems and genes in parasitic nematodes as well as plant nematodes (Meneely et al., 2019). Major technical advances, such as the cloning and physical mapping of the entire $C$. elegans genome, the development of transposon-tagging, reverse genetics, germ-line DNA transformation, genetic mosaics, and laser microsurgery, are essential to maintain and expand the usefulness of this model (Corsi et al., 2015). The most comprehensive collection of methods and information resources for analysis of $C$. elegans is found in compilation of Epstein and Shakes in 1995 (Epstein and Shakes, 1995). Knowledge gained in one area of research ultimately connects with research in other areas. Recent years have seen increasing adoption of Caenorhabditis elegans in experimental evolution in every aspects of biology.

\section{References}

Angelo, G. and Van Gilst, M. R. Starvation protects germline stem cells and extends reproductive longevity in C.elegans. $\quad$ Science. 2009; 326(5955):954-958.

Bird, D. M., Hopperman, C. H., Jones, S. J. M. and Baillie, D. L. The Caenorhabditis elegans genome: A guide in the post genomics age. Annual Review of Phytopathology. 1999;37:247-265.

Boender, A., Roubos, E. W. and Velde, G. V. Together or alone? Foraging strategies in Caenorhabditis elegans. Biological Reviews.2011;86(4):853-862.

Chalfie, M., Sulston, J. E., White, J. G., Southgate, E., Thomson, J. N. and Brenner, S. The neural circuit for touch sensitivity in Caenorhabditis elegans. Journal of Neuroscience. 1985 ;5: 956964.

Coburn, C. and Gems, D. The mysterious case of the $C$. elegans gut granule: death fluorescence, anthranilic acid and the kynurenine pathway. Frontiers in Genetics. 2013; 4 :151. doi: 10.3389/fgene.2013.00151

Corsi, A. K., Wightman, B. and Chalfie, M. A transparent window into biology: A primer on Caenorhabditis elegans. Genetics.2015; 200:387-407.

Darby, C., Hsu, J. W., Ghori, N. and Falkow, S. Caenorhabditis elegans: plague bacteria biofilm blocks food intake. Nature. 2002 ;417:243-244.

Darryl Conte Jr., Lesley, T., Mac Neil, Albertha, J. M., Walhout, C. C., Mello. RNA Interference in Caenorhabditis elegans. Current Protocols in Molecular Biology.2015;26.3.126.330.

Denzel, M. S., Lapierre, L. R. and Mack, H. I. D. Emerging topics in C. elegans aging research: Transcriptional regulation, stress response and epigenetics. Mechanisms of Ageing and Development, 2019; 177:4-21. doi: 10.1016 /j. $\operatorname{mad} .2018 .08 .001$.

Epstein, H. F. and Shakes, D. C. Caenorhabditis elegans: modern biological analysis of an organism. In: Methods in Cell Biology, (Eds, Epstein H. F. and Shakes D C). 1995; Academic Press, San Diego,654pp.

Ferris, H. and Hieb, W. F. Ellsworth C. Dougherty: A pioneer in the selection of Caenorhabditis elegans as model organism. Genetics. 2015; 200(4):9911002

Kim, K., Sato, K., Shibuya, M., Zeiger, D. M., Butcher, R. A., Ragains, J. R., Clardy, J., Touhara, K. and Sengupta, P. Two chemoreceptors mediate developmental effects of dauer 
pheromone in $C$. elegans. Science. 2009; 326:994-998.

Martinez-Finley, E. J. and Aschner, M. Revelations from the nematode Caenorhabditis elegans on the complex interplay of metal toxicological mechanisms. Journal of Toxicology. 2011. doi: 10.1155/2011/ 895236.

McGrath, P. T., Xu,Y., Ailion, M., Garrison, J. L., Butcher, R. A. and Bargmann, C. I. Parallel evolution of domesticated Caenorhabditis species targets pheromone receptor genes. Nature, $2011 ; 477: 321-325$.

Meneely, P. M., Dahlberg, C. L. and Rose, J. K. Working with worms: Caenorhabditis elegans as a model organism. Current Protocols Essential Laboratory Techniques, 2019;19, e35.doi: 10.1002/cpet.35

Mikus, H., Miller, A., Nastase, G., Serban, A., Shapira, M.and Rubinsky, B. The nematode Caenorhabditis elegans survives subfreezing temperatures in an isochoric system. Biochemical and Biophysical Research Communication. 2016;477:401-405.

Morran, L. T., Schmidt, O. G., Gelarden, I. A., Parrish, R. C. and Lively, C. M. Running with the Red Queen: Hostparasite coevolution selects for biparental sex.Science.2011 ;333:216218.

Muller, B. and Grossniklaus, U. Model organisms-A historical perspective. Journal of Proteomics. 2010; 73 (11):2054-2063
Prahlad, V., Pilgrim, D. and Goodwin, E. B. Roles for mating and environment in C. elegans sex determination. Science,2003 ;302:1046- 1049.

Rodriguez, M., Snoek, L. B., De Bono, M., Kammenga, J. E.. Worms under stress: C. elegans stress response and its relevance to complex human disease and aging. Trends in Genetics 2013 ; 29:367-374

Shen, P., Yue, Y., Zheng, J. and Park, Y. Caenorhabditis elegans: A convenient in vivo model for assessing the impact of food bioactive compounds on obesity, aging, and Alzheimer's disease. Annual Review of Food Science and Technology. 2018 ;9:1-22.

Sochova', I., Hofman, J. and Holoubek, I. Using nematodes in soil ecotoxicology. Environment International. 2006; 32:374-383

Sommer, R. J. and Bumbarger, D. J. Nematode model systems in evolution and development. Developmental Biology.2012;1(3):389-400.

Sulston, J, Du Z, Thomas K, Wilson R, Hillier L, Staden R, Halloran N, Green P, Thierry-Mieg J, Qiu L(1992). The C. elegans genome sequencing project: a beginning. Nature. 356(6364): 37-41.

White, J. G., Southgate, E., Thomson, J. N. and Brenner, S. The structure of the nervous system of the nematode Caenorhabditis elegans. Philosophical Transactions of the Royal Society of London, Series B: Biological Sciences, 1986; 314:1-340.

\section{How to cite this article:}

Gitanjali Devi. 2021. Biological Model : Caenorhabditis elegans. Int.J.Curr.Microbiol.App.Sci. 10(05): 230-235. doi: https://doi.org/10.20546/ijcmas.2021.1005.030 